\title{
Differential Impact of Ageing on Proximal and Distal Musculature in Physically Active People
}

\section{Dorota Drabarek ${ }^{1 *}$, Janusz W Błaszczyk², Janusz Jaszczuk ${ }^{3}$, Małgorzata Chalimoniuk ${ }^{1}$ and Beata Tyszkiewicz-Gromisz ${ }^{1}$}

\author{
${ }^{1}$ Józef Piłsudski University of Physical Education in Warsaw, Faculty of Tourism and Health in Biała Podlaska \\ 2Jerzy Kukuczka Academy of Physical Education, Katowice \\ ${ }^{3}$ Józef Piłsudski University of Physical Education in Warsaw, Faculty of Physical Education and Sport in Biała \\ Podlaska
}

\begin{abstract}
Age-related decline in muscular force and power is an important prognostic indicator of functional impairments in older people. Most research has focused on the quadriceps and muscles which extend and flex the elbow and ankle joints as well as handgrip strength. To date, the strength of proximal and distal muscles of the upper limbs has not been compared in groups of active adults of differing ages. The aim of the study was to compare proximal muscles - expressed as torques of extension of the arm (ArmExt) and distal muscles - expressed as handgrip strength (HGS) in young and older physically active adults. This study examined 100 physically active volunteers without neuromuscular or central-nervous system disorders: 25 older males [OM] (68.3 \pm 5.5 years) and 25 older females [OF] (67.0 \pm 5.4$)$ and a control group: 25 young males [YM] $(21.2 \pm 1.5)$ and 25 young females [YF] $(21.3 \pm 1.3)$. Strength measurements of the upper, dominant limb, were done under isometric contraction. The ArmExt measurement was done using Set Measurement Forces. For HGS measurement a hand dynamometer was used. The results showed no statistically significant differences in the ArmExt and ArmExt/kg of the OF and OM groups compared to the YF and YM groups, respectively. The HGS value for the OM group was statistically significantly lower than for the YM group. The HGS/kg in the OF and OM groups was statistically significantly lower than in the YF and YM groups, respectively too. Our results indicate a more pronounced effects of dynapenia being observed in distal muscles to compare with proximal ones in older physically active adults.
\end{abstract}

\section{Keywords}

Ageing, Proximal muscle strength, Distal muscle strength, Distal-to-proximal muscle force ratio, Physical activity

\section{Introduction}

Introduced recently term "dynapenia" is applied to describe the age-related loss of muscle strength [1]. It has been well documented that age-related decline in muscular force and power is an import- ant prognostic indicator of functional impairments in older people [2]. In older adults reduced muscle strength involves serious consequences due to the increased difficulty of performing everyday tasks, progressive physical and functional disabilities and

*Corresponding author: Dorota Drabarek, Faculty of Tourism and Health in Biała Podlaska, Józef Piłsudski University of Physical Education in Warsaw, 2 Akademicka Str, 21-500 Biała Podlaska, Poland

Accepted: July 09, 2019; Published: July 11, 2019

Copyright: (C 2019 Drabarek D, et al. This is an open-access article distributed under the terms of the Creative Commons Attribution License, which permits unrestricted use, distribution, and reproduction in any medium, provided the original author and source are credited.

Drabarek et al. Int J Biomech Mov Sci 2019, 1:001 
limitations [3-5] as well as the decline in postural stability associated with increased risk of falling [610] and even death [11]. Therefore, it is crucial for older individuals and physiotherapists to become aware of the importance of muscle strength.

To date, most research has focused on the quadriceps and muscles which extend and flex the elbow and ankle joints as well as handgrip strength [12]. It has been found that annually the muscle strength of older individuals declines by 1-2\% [13] in both distal and proximal muscles [12]. However, in these studies the muscles of the shoulder were not taken into consideration.

So far little research has been done on the strength of shoulder muscles in older individuals. The strength of proximal muscles, those of the shoulder, and distal muscles of the upper limb in physically active individuals but differing in age has not yet been compared.

The term "proximal muscles" refers to the group of muscles responsible for extending the shoulder joint, lying close to the vertical axis of the torso. The group of muscles responsible for handgrip, anatomically farther from the vertical axis of the trunk, is known as "distal".

The main aim of the study was to compare proximal muscles - expressed as torques of extension of the arm (ArmExt) and distal muscles - expressed as handgrip strength (HGS) in young and older physically active adults. We posit that the process of dynapenia might differentially affect the proximal and distal musculature.

\section{Methods}

The study was carried out in 2013 and 2014 in the Academy of Physical Education in Biala Podlas$\mathrm{ka}$, Poland. The study was designed in accordance with the Declaration of Helsinki and was approved by the Senate Ethics Committee for Scientific Research, Josef Piłsudski Academy of Physical Educa- tion in Warsaw, Poland. All subjects signed an informed consent for participation in the study.

\section{Participants and recruitment}

The study involved 100 individuals; 50 older adults (60+ years old): 25 older females (OF) and 25 older males (OM); 50 young adults (20 to 25 -yearsold): 25 young females (YF) and 25 young males (YM). The control group was made up of young adults. Only able-bodied subjects were included in the study who for at least one year had regularly participated in some type of physical activity twice a week (45 $\mathrm{min}$ ) such as aerobics, swimming, Nordicwalking or biking. During the month before the study began each person was interviewed in order to determine his/her health and level of physical activity. Individuals with motor function diseases (for example: Rheumatoid arthritis) and diseases of the nervous system (for example: Stroke, Parkinson's and multiple sclerosis) and hypertension were eliminated. Before the study began blood pressure at rest was taken in the older adults. Individuals with a blood pressure equal to or greater than 140/90 mmHg were excluded from the study. Height and weight were also measured. Characteristics of the study are presented in Table 1.

\section{Muscle strength measurements}

Muscle strength measurements were done on the upper limb which the participant declared was functionally dominant. Isometric contractions were measured according to Tokarski, et al. [14] with slight modifications. During measurements the participants stood with trunk and head straight. The angles of the upper extremity were $0^{\circ}$ for the arm, $0^{\circ}$ for the elbow and $0^{\circ}$ for the angle between the forearm and the hand. Before measurements were made the participants did some standard warm-up exercises of the muscles to be studied. One trial test prior to the measurements were also done. For each individual three muscle strength measurements were done for proximal and distal

Table 1: Characteristics of the research groups (mean \pm SD).

\begin{tabular}{|l|l|l|l|l|l|}
\hline Group & Age (yrs) & Height $(\mathbf{c m})$ & Body mass $\mathbf{( k g )}$ & BMI & Systematic physical activity \\
\hline YF (n= 25) & $21.3 \pm 1.3$ & $170.0 \pm 6.3$ & $61.1 \pm 1.17$ & $21.32 \pm 2.82$ & $2 \times /$ week \\
\hline YM (n= 25) & $21.2 \pm 1.5$ & $182.0 \pm 7.4$ & $78.2 \pm 1.20$ & $23.87 \pm 3.65$ & $2 \times /$ week \\
\hline OF (n= 25) & $67.0 \pm 5.4$ & $162.3 \pm 4.5$ & $69.7 \pm 1.16$ & $26.72 \pm 3.51$ & $2 \times /$ week \\
\hline OM (n= 25) & $68.3 \pm 5.5$ & $173.9 \pm 5.2$ & $82.9 \pm 1.1$ & $27.71 \pm 3.48$ & $2 \times /$ week \\
\hline
\end{tabular}

YF: Young Female; YM: Young Male; OF: Older Female; OM: Older Male. 
muscles. Between each measurement there was a one minute break to avoid muscle fatigue. For further analysis the highest value of each trial was used. The trials were done in the morning, 2-3 hours after standard breakfast.

\section{Proximal muscle strength measurements}

Force and its arm of action were measured to assess ArmExt. Force measurements were done with the Measurement Force Kit ZPS4-P (JPA Zb. Staniak, Warsaw, Poland).

\section{Distal muscle strength measurements}

HGS measurements were done with handgrip dynamometer DR 5 (JBA Zb. Staniak - Warsaw, Poland). The variable width of the dynamometer grip made it possible to adjust the distance between the levers, depending on the size of the participant's arms.

\section{Statistical analysis}

All statistical analyses were performed using Statistica v. 12.0 software (Statsoft, Tulsa, OK, USA).
Conformity of variables with normal distribution was tested with the Shapiro-Wilk test. One-way analysis of variance was used in order to examine statistically significant differences in the analyzed parameters. Detailed comparisons were made using Tukey's post-hoc test. The power of the test was calculated with the assumption of an error type I alpha $=0.05$. Association between variables (HGS, $\mathrm{HGS} / \mathrm{kg}$, ArmExt, ArmExt/kg and BMI) was evaluated with the Pearson's correlation test. In all cases, a $p<0.05$ was considered significant.

\section{Results}

Anthropometric data of participants were shown in Table 1.

\section{Differences in muscle strength between groups of men and women}

Statistically significant higher values were found in men than in women for all strength measurements of both age groups: ArmExt (Nm), ArmExt standardized for body weight (ArmExt/kg) (Nm/ $\mathrm{kg}), \mathrm{HGS}(\mathrm{N})$ and HGS standardized for body weight
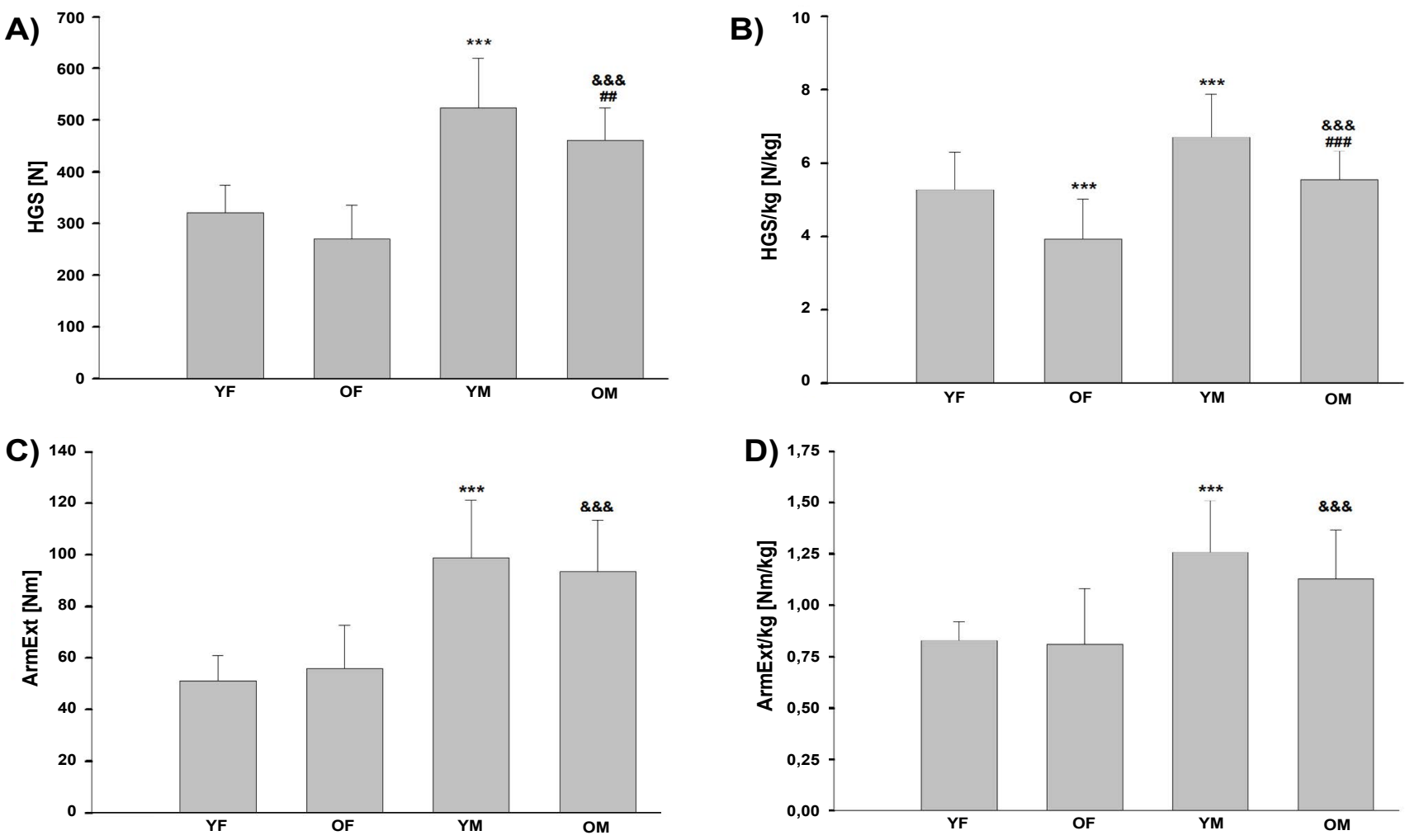

Figure 1: Values (mean $\pm S D$ value) of distal $(A$ and $B$ ) and proximal ( $C$ and $D)$ muscle strength of the upper limb of older and young physically active adults.

YF: Young Female; OF: Older Female; YM: Young Male; OM: Older Male; HGS: Handgrip Strength; ArmExt: Torques of Extension of the Arm; N: Newton; Nm: Newton Meter; ${ }^{* * *} p<0.001$ versus YF; \#\#p $<0.01$, \#\#\#p $<0.001$ versus YM; \&\&\&p $<0.001$ versus OF. 
(HGS/kg) (N/kg) ( $\mathrm{p} \leq 0.001)$. Therefore, muscle strength values were compared for the age groups of the same gender (Figure 1). Statistical analysis showed that the power of the test with assumed error type I alpha $=0.05$ was 1 .

\section{Proximal muscle strength}

The ArmExt value in the OF group was higher than in the YF group and in the OM group it was lower than that of the YM group. There were lower ArmExt/kg values for the OF and OM than for the YF and YM groups, respectively. This difference however was not statistically significant (Figure 1). Statistical analysis showed that the power of the test with assumed error type I alpha $=0.05$ was 1 . Statistical analysis indicated positive correlation between ArmExt and BMI ( $r=0.321)$.

\section{Distal muscle strength}

The HGS value for the OM group was statistically significantly lower than for the YM group by $12 \%$ ( $p \leq 0.01$ ), while in the OF group there was a tendency for lower HGS values $(p \leq 0.08)$ than in the YF group. The HGS/kg value in the OF group was statistically significantly lower than the YF group by $26 \%$ ( $p \leq 0.001)$, and in the OM group it was lower than the YM group by $17 \%$ ( $p \leq 0.001$ ) (Figure 1 ). Statistical analysis showed that the power of the test with assumed error type I alpha $=0.05$ was 1 . Statistical analysis indicated negative correlation between $\mathrm{HGS} / \mathrm{kg}$ and $\mathrm{BMI}(r=-0.458)$.

\section{Discussion}

A comparison was made between the strength of muscles which extend the shoulder joint and handgrip strength. The study compared two sizeable $(n=50)$ groups of physically active older adults $(60+)$ and young individuals. In contrast to young individuals' older persons had significantly lower distal muscle strength: HGS and HGS/kg (a tendency for lower HGS values was seen in women). Both groups, however, had similar values for proximal muscle strength: ArmExt and ArmExt/kg.

Previous research reported a similar age-related decline of the proximal and distal limb muscle strength in older individuals. However, these reports dealt only with muscles responsible for handgrip and the knee, ankle and elbow joints and did not determine the muscle strength of the shoulder joint [12]. We found that older, physically active individuals had less handgrip muscle strength, but not less strength in the muscles extending the shoulder joint.

The results of our research agree with the observations of Galea [15], namely that the consequences of ageing are different for distal and proximal muscles. Galea [15], after determining the numbers of motor units in the thenar muscles, in the extensor digitorum brevis and in the biceps brachii of healthy people 20 to 98 -years-old, found a significant loss in older individuals of the distal muscles. Galea [15] suggested, however, that the biceps brachii did not change. Of the muscles responsible for extending the shoulder joint, the triceps brachii - Caput longum corresponds to the biceps brachii. The remaining muscles which extend the shoulder joint: - The deltoideus - pars spinalis, the teres major muscle and the latissimus dorsi are even more internal and so it seems that they should change even less. However, Raz, et al. [16] using magnetic resonance imaging with arthrography -MRA, documented the age-related decrease in cross sectional areas of the deltoideus, supraspinatus, infraspinous, teres minor and subscapularis muscles in 249 patients who had no tearing of these muscles. These researchers concentrated on only five of the mentioned muscles, which did not make it possible to describe changes in the distal muscles. Furthermore, atrophy of the mentioned muscles in older individuals can result from smaller size individual myofibers while maintaining the same number of motor units. Raz, et al. [16] did not include information about how much physical activity the subjects usually had. If there was a low level of physical activity it is natural that during the ageing process, there is a loss of muscle mass which can cause loss of strength. This may result from what has already been shown that there is a positive relationship between the size of a cross sectional area of muscle and its strength [17]. Since all the subjects of our study had systematic level of physical activity the lack of change in the numbers of motor units near the shoulder joint of older persons could explain why their proximal muscle strength was comparable to younger persons. This agrees with Galea's research [15].

The results of Tokarski, et al. [14] differ from our results and show significantly lower values of muscles that extend the arm at the shoulder joint in older persons than in those who are young. In the cited studies, as well as ours, dynometers made by 
JBA Staniak (Poland) were used. However, it needs to be emphasized that people participating both in Tokarski's and our research were in different positions while being measured (Tokarski, et al. [14]: $0^{\circ}$ for the arm, $90^{\circ}$ for the elbow and $0^{\circ}$ for the hand; our study: $0^{\circ}$ for the arm, $0^{\circ}$ for the elbow and $0^{\circ}$ for the hand). But muscle force generation is posture dependent [18]. According to RomanLiu and Tokarski [19] the maximum strength of the upper limbs depends on the position of the limb during measurement. In the study being referred to the position was different for each measurement. This makes it difficult to assess the real differences. It is necessary to determine the influence of age on the muscular strength of the shoulder with a uniform, standardized starting position.

Also, Vidt, et al. [20] compared muscle strength at the shoulder (abduction and adduction), elbow (flexion and extension), and wrist (flexion and extension) of the elderly with young and found in the elderly the lower muscle strength for all joints with significant differences for shoulder abductors, adductors and wrist flexors. They did not find statistically significant differences in the strength of elbow flexors, extensors and wrist extensors. The authors explain that observed differences may result from greater involvement of the wrist and elbow muscles than the shoulders muscles in everyday activities $[20,21]$.

The research results presented by us, in contrast to the above, have shown that elderly people maintain shoulder extensors at a level comparable to that of young people. It is also significant that only in our study all participants were systematically physically active. In the study of Tokarski, et al. [14] the participants had a low level of physical activity during their free time. Participants of Vidt, et al.) [20] research were defined only as healthy people.

It has been well documented that physical activity and exercise counteracts age-related decline in muscle mass, strength, and muscle regenerative capacity [22] that in turn has positive impact on physical performance (gait speed, chair rising test, balance, etc) [23]. According to Descheres, et al. [24] and Gonzales-Freire, et al. [25] a high level of neuromuscular activity may delay and attenuate the onset of age-related progressive impairment of motor function. It can be supposed that regular physical exercise by older people in our study helped maintain proximal muscle strength at a level similar to young persons.

We found that the handgrip strength was dependent on gender and also was significantly lower in older subjects to compare with younger individuals. This is in agreement with earlier studies [14,26-31].

Weaker handgrip in older persons was also found by Tokarski, et al. [14] in a group of women aged 20-62. Rantanen, et al. [26] reported that handgrip strength weakened together with age in a group of men aged 45-68. The measurements were repeated on those who were still alive 27 years later. Lauretani, et al. [27] and Kallman, et al. [28] studied women and men in a wide age range from 20 to 102 and found that handgrip weakened with ageing processes. Su, et al. [29] studying 80 women and 80 men aged 20 to 69 had similar results. In agreement with the above studies Han, et al. [30] in their research on 235 men aged 10-74 and 280 women, aged 14-84, made similar observations. Skelton, et al. [13] and Jansen, et al. [31] showed that handgrip strength consistently decreased with age, even when comparing only older individuals. This was seen in their research which was done on 100 women and men aged $65-89$ as well as 224 women and men aged 65-92.

The results of our comparison of the handgrip strength of women and men are in accordance with Lauretani, et al. [27] and Han, et al. [30]. These researchers reported that in all age groups men had stronger handgrips than women. The results of studies on people over 65 done by Bassey and Harries [32], Skelton, et al. [13], Rantanen, et al. [33], Al Snih, et al. [34] as well as Jansen, et al. [31] also confirm this fact for groups of older individuals.

In summary, our results indicate a more pronounced effects of dynapenia being observed in distal muscles to compare with proximal ones in regularly physically active women and men $60+$. Both, the older women and men had significantly lower handgrip strength while, at the same time, the strength of their muscles that extend the arm at the shoulder joint was comparable to that observed in young individuals. These results can suggest that systematic physical activity (aerobics, swimming, Nordic-walking or biking) in older persons may delay the onset of muscle strength loss in proximal muscles but not that of distal muscles. 
The results of the research may be used in physiotherapeutic programs aimed at maintaining proximal muscle strength in the upper limbs, which may be important in the prevention of disability and loss of independence of older people.

\section{Acknowledgement}

The work has been prepared under the research project of the Faculty of Physical Education and Sport in Biała Podlaska, Józef Piłsudski University of Physical Education in Warsaw - DS. 194 - financed by the Ministry of Science and Higher Education.

\section{References}

1. Clark BC, Manini TM (2008) Sarcopenia $\neq$ dynapenia. J Gerontol A Biol Sci Med Sci 63: 829-834.

2. Clark BC, Manini TM (2012) What is dynapenia? Nutrition 28: 495-503.

3. Hairi NN, Cumming RG, Naganathan V, Handelsman DJ, Le Couteur DG, et al. (2010) Loss of muscle strength, mass (sarcopenia), and quality (specific force) and its relationship with functional limitation and physical disability: The concord health and ageing in men project. J Am Geriatr Soc 58: 2055-2062.

4. Ploutz-Snyder LL, Manini T, Ploutz-Snyder RJ, Wolf DA (2002) Functionally relevant thresholds of quadriceps femoris strength. J Gerontol A Biol Sci Med Sci 57: 144-152.

5. Manini TM, Visser M, Won-Park S, Patel KV, Strotmeyer ES, et al. (2007) Knee extension strength cutpoints for maintaining mobility. J Am Geriatr Soc 55: 451-457.

6. Horlings CG, Van Engelen BG, Allum JH, Bloem BR (2008) A weak balance: The contribution of muscle weakness to postural instability and falls. Nat Clin Pract Neurol 4: 504-515.

7. Schöne D, Freiberger E, Sieber CC (2017) Influence of skeletal muscles on the risk of falling in old age. Der Internist 58: 359-370.

8. Melzer I, Benjuya N, Kaplanski J, Alexander N (2008) Association between ankle muscle strength and limit of stability in older adults. Age Ageing 38: 119123.

9. Carter ND, Khan KM, Mallinson A, Janssen PA, Heinonen A, et al. (2002) Knee extension strength is a significant determinant of static and dynamic balance as well as quality of life in older communitydwelling women with osteoporosis. Gerontology 48: 360-368.

10. Matsumura BA, Ambrose AF (2006) Balance in the elderly. Clin Geriatr Med 22: 395-412.
11. Newman AB, Kupelian V, Visser $M$, Simonsick EM, Goodpaster BH, et al. (2006) Strength, but not muscle mass, is associated with mortality in the health, aging and body composition study cohort. J Gerontol A Biol Sci Med Sci 61: 72-77.

12. Doherty TJ (2003) Invited review: Aging and sarcopenia. J Appl Physiol 95: 1717-1727.

13. Skelton DA, Greig CA, Davies JM, Young A (1994) Strength, power and related functional ability of healthy people aged 65-89 years. Age and Aging 23: 371-377.

14. Tokarski T, Roman-Liu D, Kamińska J (2012) The influence of age and type of force on muscle strength capabilities in women. Int J Occup Saf Ergon 18: 47-57.

15. Galea V (1996) Changes in motor unit estimates with aging. J Clin Neurophysiol 13: 253-260.

16. Raz $Y$, Henseler JF, Kolk $A$, Riaz $M$, van der Zwaal $P$, et al. (2015) Patterns of age-associated degeneration differ in shoulder muscles. Front Aging Neurosci 7: 236.

17. Narici MV, Maffulli N (2010) Sarcopenia: Characteristics, mechanisms and functional significance. Br Med Bull 95: 139-159.

18. Zajac FE (1989) Muscle and tendon: Properties, models, scaling, and application to biomechanics and motor control. Crit Rev Biomed Eng 17: 359411.

19. Roman-Liu D, Tokarski T (2005) Upper limb strength in relation to upper limb posture. Int J Ind Ergon 35: 19-31.

20. Vidt ME, Daly M, Miller ME, Davis CC, Marsh AP, et al. (2012) Characterizing upper limb muscle volume and strength in older adults: A comparison with young adults. J Biomech 45: 334-341.

21. Landers KA, Hunter GR, Wetzstein CJ, Bamman MM, Weinsier RL (2001) The interrelationship among muscle mass, strength, and the ability to perform physical tasks of daily living in younger and older women. J Gerontol A Biol Sci Med Sci 56: 443-448.

22. Distefano G, Goodpaster BH (2018) Effects of exercise and aging on skeletal muscle. Cold Spring Harb Perspect Med 8: 1-16.

23. Beaudart C, Dawson A, Shaw SC, Harvey NC, Kanis JA, et al. (2017) Nutrition and physical activity in the prevention and treatment of sarcopenia: Systematic review. Osteoporos Int 28: 1817-1833.

24. Deschenes MR, Roby MA, Eason MK, Harris MB (2010) Remodeling of the neuromuscular junction 
precedes sarcopenia related alterations in myofibers. Exp Gerontol 45: 389-393.

25. Gonzalez-Freire M, de Cabo R, Studenski SA, Ferrucci $L$ (2014) The neuromuscular junction: Aging at the crossroad between nerves and muscle. Front Aging Neurosci 6: 208.

26. Rantanen T, Masaki K, Foley D, Izmirlian G, White L, et al. (1998) Grip strength changes over $27 \mathrm{yr}$ in Japanese-American men. J Appl Physiol 85: 20472053.

27. Lauretani F, Russo CR, Bandinelli S, Bartali B, Cavazzini C, et al. (2003) Age-associated changes in skeletal muscles and their effect on mobility: An operational diagnosis of sarcopenia. J Appl Physiol 95: 1851-1860.

28. Kallman DA, Plato CC, Tobin JD (1990) The role of muscle loss in the age-related decline of grip strength: Cross-sectional and longitudinal perspectives. J Gerontol 45: 82-88.

29. Su CY, Lin JH, Chien TH, Cheng KF, Sung YT (1994) Grip strength in different positions of elbow and shoulder. Arch Phys Med Rehabil 75: 812-815.

30. Han SH, Nam KS, Cho YS, Ryu KJ (2011) Normative data on hand grip strength. J Nov Physiother 1: 102.

31. Jansen CWS, Niebuhr BR, Coussirat DJ, Hawthorne D, Moreno L, et al. (2008) Hand force of men and women over 65 years of age as measured by maximum pinch and grip force. J Aging Phys Activity 16: 24-41.

32. Bassey EJ, Harries UJ (1993) Normal values for handgrip strength in 920 men and women aged over 65 years, and longitudinal changes over 4 years in 620 survivors. Clin Sci (Lond) 84: 331-337.

33. Rantanen $T$, Era $P$, Kauppinen M, Heikkinen $E$ (1994) Maximal isometric muscle strength and socioeconomic status, health, and physical activity in 75-year-old persons. J Aging Phys Activity 2: 206220.

34. Al Snih S, Markides KS, Ottenbacher KJ, Raji MA (2004) Hand grip strength and incident ADL disability in elderly Mexican Americans over a seven-year period. Aging Clin Exp Res 16: 481-486. 